\title{
Genetic suppression analysis of non-antibiotic- producing mutants of the Streptomyces coelicolor absA locus
}

\author{
Todd Anderson, Paul Brian, $\uparrow$ Perry Riggle, $\neq$ Renqiu Kong \\ and Wendy Champness
}

Department of

Microbiology, Michigan State University, East Lansing, MI 48824-1101, USA

\author{
Author for correspondence: Wendy Champness. Tel: +1 517353 9770. Fax: +1 5173538957. \\ e-mail: champnes@pilot.msu.edu
}

Keywords: Streptomyces coelicolor, antibiotic biosynthesis and regulation, twocomponent system, genetic suppression

\section{INTRODUCTION}

Actinomycetes produce most of the antibiotics useful for medical treatments and the genus Streptomyces contains an especially large number of antibiotic-producing species (Strohl, 1997). Streptomyces coelicolor has been the species of choice for genetic investigation of fundamental questions about antibiotic synthesis and regulation (Champness \& Chater, 1994). Like other streptomycetes, S. coelicolor produces antibiotics in a growthphase-dependent manner (reviewed by Bibb, 1996). In liquid cultures, antibiotics are produced only after the rapid growth phase. Antibiotic production is also

† Present address: TerraGen Diversity Inc., Vancouver, British Columbia, Canada.

¥Present address: Department of Molecular Biology and Microbiology, Tufts University, Boston, MA 02111, USA.

Abbreviations: Act, actinorhodin; CDA, calcium-dependent antibiotic; Mmy, methylenomycin; NF, normal fertility; Red, undecylprodigiosin; UF, ultra fertility. delayed in surface cultures, where it occurs in conjunction with sporulation.

In S. coelicolor, coupling of antibiotic production to sporulation is easily observed visually because two of the four antibiotics produced are pigments. Actinorhodin (Act) is blue or red, depending on $\mathrm{pH}$, and undecylprodigiosin (Red) is red or yellow, its colour also varying with $\mathrm{pH}$. Early genetic studies, using colour phenotypes to define the genes required for Act and Red synthesis (Rudd \& Hopwood, 1979, 1980), showed that these genes comprised two clusters, act and red, respectively. The other two S. coelicolor antibiotics, methylenomycin (Mmy) and calcium-dependent antibiotic (CDA), have also been genetically characterized (Chater \& Bruton, 1985; Chong et al., 1998).

Molecular characterization of the antibiotic gene clusters has included cloning, sequencing, definition of transcripts and identification of cluster-specific regulatory genes. The act cluster consists of at least six transcripts and 20 ORFs. The act- and red-specific 
regulators [ActII-ORF4 (Fernández-Moreno et al., 1991; Gramajo et al., 1993) and RedD (Takano et al., 1992; Narva \& Feitelson, 1990)] are the best understood. They are both activators with considerable amino acid sequence similarity and are members of the growing SARP family (streptomycete antibiotic regulatory protein) of transcriptional regulators (Wietzorrek \& Bibb, 1997).

Growth-phase-regulated expression of these antibioticspecific regulators is an important component of antibiotic regulation (Bibb, 1996). Approaches to defining the genetic mechanisms responsible (reviewed by Champness, 1999a) have included analysis of cloned genes that enhance antibiotic production, evaluation of the roles of metabolic regulators such as relA through gene disruption (e.g. Chakraburtty \& Bibb, 1997), and screening for mutations that perturb antibiotic regulation (reviewed by Champness, 1999b; Chater \& Bibb, 1997).

In one genetic analysis of $S$. coelicolor antibiotic regulation, some mutations that blocked production of all four known antibiotics ( $\mathrm{Abs}^{-}$phenotype, for antibiotic synthesis deficient) were found to define the abs $\bar{A}$ locus (Adamidis et al., 1990). This locus was subsequently shown to encode a two-component signal transduction system (Brian et al., 1996) composed of AbsA1, a homologue of 'orthodox' histidine kinase sensor-transmitter proteins, and AbsA2, a putative DNA-binding response regulator with the amino acid sequences conserved in 'orthodox' response-regulator proteins (Parkinson, 1995; Stock et al., 1995). The abs A locus regulates transcription of the actII-ORF4 and redD pathway-specific activators (Aceti \& Champness, 1998).

The absA mutants isolated on the basis of their $\mathrm{Abs}^{-}$ phenotype were shown, by marker rescue experiments (Brian et al., 1996), to carry mutations of the absA1 gene. In contrast, disruptions of the locus resulted in a phenotype of early onset enhanced antibiotic production. This phenotype was associated with disruption of both $a b s A 1$ and $a b s A 2$ or of $a b s A 2$ alone. Hence, we proposed (Brian et al., 1996) that the absA locus exerts a negative regulatory effect on antibiotic production and that the $\mathrm{Abs}^{-}$abs A strains are mutationally locked into the negatively acting state.

Two Abs abs A mutant strains, C542 and C577, were phenotypically characterized in detail. One shared trait is spontaneous apparent reversion. We were interested in determining the genetic events underlying formation of the apparent revertants because if these strains contained suppressive mutations, the suppressors might provide insights into the mechanisms of AbsA1/AbsA2 function or identify other genetic elements functioning in the abs A pathway.

Here, we present a genetic analysis of the abs $A$ pseudoreversion phenomenon. We first define the absA1 mutational alterations in $\mathrm{Abs}^{-}$strains and then demonstrate that a set of pseudorevertant strains carries second-site suppressive mutations, sab (for suppressor of $\underline{a b} s A$ ). We use plasmid-mediated and protoplast fusion mapping techniques to locate $s a b$ alleles and then use a specialized transducing phage to show that some $s a b$ mutations map to the abs A locus. By sequence analysis, we define the mutational alterations to the AbsA1 and AbsA2 proteins.

\section{METHODS}

Bacterial and phage strains. Derivatives of $S$. coelicolor A3(2) and phage strains are listed in Table 1. Streptomyces lividans 1326 was used for phage propagation. Escherichia coli DH5 $\alpha$ (Gibco-BRL) was used for plasmid cloning. S. coelicolor and E. coli culture techniques were as previously described (Brian et al., 1996).

Culture conditions for antibiotic assays. R5 agar medium (Hopwood et al., 1985) was used for characterization of Act, Red and Mmy. SMMS agar (Takano et al., 1992) was also used for Act and Red, which were assayed as described previously (Brian et al., 1996). Mmy assays were as described by Brian et al. (1996). For assay of CDA, low-calcium nutrient agar (Oxoid) with or without added calcium $\left[\right.$ as $\mathrm{Ca}\left(\mathrm{NO}_{3}\right)_{2}$ to $12 \mathrm{mM}$ ] was used (Adamidis et al., 1990).

Isolation of sab strains. Pigmented strains were cultured from spore to spore a minimum of three times and then acid-treated (0.01 $\mathrm{M} \mathrm{HCl}, \mathrm{pH} 2 \cdot 0$, for $10 \mathrm{~min})$, followed by neutralization with an equal volume of $0 \cdot 01 \mathrm{M} \mathrm{NaOH}$ to kill any contaminating mycelia that might carry multiple genomes, and therefore carry a $s a b^{+}$allele.

$s a b$ strains were isolated from spontaneously arising pigmented sectors in plates of strains C542 and C577. To ensure that independent $s a b$ mutations were isolated, $\mathrm{Abs}^{-}$spores were plated and the resulting individual colonies were cultured through several rounds of streaking before pigmented sectors were selected. Each $s a b$ strain was isolated from a different $\mathrm{Abs}^{-}$progenitor colony.

Genetic mapping techniques. Crosses and data analysis were carried out as described previously (Champness, 1988). For plasmid-mediated mating, chromosomal recombination was mediated primarily by plasmid SCP1 integrated at 9 o'clock on the genetic map to give the NF type (Hopwood \& Chater, 1974). In an $\mathrm{NF} \times \mathrm{SCP}^{-}$cross (also referred to as $\mathrm{NF} \times \mathrm{UF}$ ), close to $100 \%$ of the progeny will be NF (Hopwood et al., 1969). Several phenotypes are associated with the NF state: (1) $\mathrm{NF}$ strains are $\mathrm{Aga}^{-}$and fail to sink into the agar whereas $\mathrm{Aga}^{+}$ strains sink (Hodgson \& Chater, 1981); and (2) NF strains (including $\mathrm{Abs}^{-}$strains) are usually Mmy-resistant due to the presence of the mmy gene cluster on SCP1 (Kirby \& Hopwood, 1977).

The frequencies of markers donated by the NF parent decrease in both clockwise and anticlockwise directions from the SCP1 insertion region at 9 o'clock (Hopwood et al., 1985). If the $\mathrm{SCP}^{-}$parent is $\mathrm{J} 1501$ (Table 1), the his 11 and strA1 alleles will be present in $>95 \%$ of spore progeny plated nonselectively.

Protoplast fusions. PEG-mediated protoplast fusions were performed as described by Hopwood et al. (1985). Spores from regenerated protoplasts were plated on selective media.

Actinophage transductions. Transductions were carried out essentially as described by Piret \& Chater (1985). Briefly, 
Table 1. S. coelicolor A3(2) strains and Streptomyces phage

\begin{tabular}{|c|c|c|}
\hline \multicolumn{2}{|r|}{ Relevant characteristics } & \multirow[t]{2}{*}{ Source or reference } \\
\hline Strains & & \\
\hline J1501 & hisA1 uraA1 strA1 $\mathrm{SCP}^{-} \mathrm{SCP}^{-} \mathrm{Pgl}^{-}$ & Hopwood et al. (1985) \\
\hline $\mathrm{J} 650$ & cysD18 mthB2 SCP1 $1_{\mathrm{NF}} \mathrm{SCP} 2 *$ & Hopwood et al. (1985) \\
\hline 1514 & cys $A 15$ proA1 argA1 uraA1 nicA1 SCP1 $1_{\mathrm{NF}} \mathrm{SCP} 2 *$ & Hopwood et al. (1985) \\
\hline TK16 & $\arg A 1$ guaA1 redA59 act-117 (IV) & Hopwood et al. (1985) \\
\hline TK18 & $\arg A 1$ uraA1 strA1 redE60 act-141 (III) & Hopwood et al. (1985) \\
\hline $\mathrm{C} 420$ & $\triangle a b s A 1:$ ermE hisA1 uraA1 strA1 $\mathrm{SCP}^{-} \mathrm{SCP}^{-} \mathrm{Pgl}^{-}$ & Brian et al. (1996) \\
\hline $\mathrm{C} 430$ & $\triangle a b s A 2:$ : RS500 hisA1 uraA1 strA1 $\mathrm{SCP}^{-} \mathrm{SCP}^{-} \mathrm{Pgl}^{-}$ & Brian et al. (1996) \\
\hline M138 & $\arg A 1$ cysD18 proA1 $\mathrm{SCP} 1_{\mathrm{NF}}$ & Hopwood et al. (1985) \\
\hline C542 & his 1 1 uraA1 strA1 absA1-542 $\mathrm{SCP}^{-} \mathrm{SCP}^{-} \mathrm{Pgl}^{-}$ & Adamidis et al. (1990) \\
\hline C577 & hisA1 uraA1 strA1 absA1-577 $\mathrm{SCP}^{-} \mathrm{SCP}^{-} \mathrm{Pgl}^{-}$ & Adamidis et al. (1990) \\
\hline C5422 & cys 115 proA1 argA1 uraA1 absA1-542 SCP1 ${ }_{\mathrm{NF}} \mathrm{SCP} 2 *$ & Adamidis et al. (1990) \\
\hline C5423 & cysD18 mthB2 absA1-542 SCP1 $1_{\mathrm{NF}} \mathrm{SCP} 2 *$ & This work $\dagger$ \\
\hline C5422S1 & C5422 sab1, Type II & This work \\
\hline C542S2 & C542 sab2, Type II & This work \\
\hline $\mathrm{C} 542 \mathrm{~S} 3$ & C542 sab3, Type I & This work \\
\hline C542S9 & C542 sab9, Type II & This work \\
\hline C542S10 & C542 sab10, Type I & This work \\
\hline C542S11 & C542 sab11, Type II & This work \\
\hline C542S12 & C542 sab12, Type II & This work \\
\hline C577S20 & C577 sab20, Type I & This work \\
\hline C577S25 & C577 sab25, Type II & This work \\
\hline C577S27 & C577 sab27, Type I & This work \\
\hline C577S28 & C577 sab28, Type II & This work \\
\hline \multicolumn{3}{|l|}{ Phage } \\
\hline KC516 & $\phi$ C31-derived actinophage, Thio ${ }^{\mathrm{r}}$ att $^{-} c^{+}$ & Rodicio et al. (1985) \\
\hline RS100 & KC516 carrying $3 \cdot 2 \mathrm{~kb}$ XhoI insert & Brian et al. (1996) \\
\hline RS120 & KC516 carrying abs A1-542 mutant $3 \cdot 2 \mathrm{~kb}$ XhoI insert & Brian et al. (1996) \\
\hline RS500 & KC516 carrying $1.45 \mathrm{~kb}$ XhoI-Bam HI insert & Brian et al. (1996) \\
\hline
\end{tabular}

† Recombinant from cross of C542 × J650 strains.

phage suspensions were mixed with spores of the recipient strain on R5 agar. After colonies had sporulated, they were replicated to $\mathrm{R} 5$ agar containing thiostrepton $\left(50 \mu \mathrm{g} \mathrm{ml}^{-1}\right)$ to select lysogens. Phage released from lysogens were isolated as plaques formed in S. lividans soft agar overlays poured on to 1 -d-old lysogenic cultures streaked on nutrient agar. Nutrient agar and phage propagation techniques were those described by Hopwood et al. (1985).

DNA sequencing of absA1 and sab mutant alleles. The $a b s A 1$ alleles of $\mathrm{Abs}^{-}$strains $\mathrm{C} 542$ and C577 and the presumptive $s a b$ mutations were sequenced from nested PCR-generated fragments. The absA1 gene (Brian et al., 1996) was amplified from primers A15a (5'-CATCTGGCGGCGATCGGCAACGACCG-3'; nt 54-71 upstream of the absA1 translation start site) and A13e (5'-CGCGAATCATCCGATCGTTCCCTGGTG$3^{\prime}$; nt 2-28 downstream of the absA1 stop codon). The PCR reaction mixtures $(100 \mu \mathrm{l})$ contained $200 \mathrm{ng}$ genomic DNA template, $0.3 \mu \mathrm{mol}$ of each primer and $2.5 \mathrm{U}$ Pfu polymerase (Stratagene). Template denaturation for $5 \mathrm{~min}$ at $95^{\circ} \mathrm{C}$ was followed by 30 cycles of $95^{\circ} \mathrm{C}$ for $1 \mathrm{~min}, 65^{\circ} \mathrm{C}$ for $1.5 \mathrm{~min}$ and $72{ }^{\circ} \mathrm{C}$ for $1 \mathrm{~min}$, and a final extension at $72^{\circ} \mathrm{C}$ for $10 \mathrm{~min}$. The $1.8 \mathrm{~kb}$ abs 11 product was purified by agarose gel electrophoresis, digested with BamHI and XhoI, and cloned in pBluescript II SK $(+)$ (Stratagene). Because previous genetic mapping had localized the absA1 mutations to the $1.45 \mathrm{~kb}$ XhoI-BamHI segment of absA1 (Brian et al., 1996), sequence analysis was restricted to this region. The end regions were sequenced from standard vector primers, while the internal region was sequenced from primer A15c (5'-CGCTACATCGCCGACCAG-3'; nt 546-563 of absA1) (Iowa State University DNA Sequencing Facility, Ames, IA, USA). The absA1 Xhol/BamHI regions of sab mutants C542S3 and C542S11 were sequenced in the same manner. In addition, the $\mathrm{N}$ - and C-termini of these alleles were confirmed by direct sequencing of the PCR product using primers A15a and A13e.

The $s a b$ absA2 sequences were generated with primers A25a (5'-CAGGGAAGGATCCGATGATTCGCG-3'; nt -18 to +10 of $a b s A 2)$ and A23a (5'-GGGCGACCGGCGGATCCGCCTC-3'; nt 80-101 downstream of the absA2 stop codon), both of which contain BamHI restriction sites. Each reaction mixture $(100 \mu \mathrm{l})$ contained $200 \mathrm{ng}$ genomic DNA template, $0.3 \mu \mathrm{mol}$ of each primer and $2.5 \mathrm{U}$ Taq polymerase (GibcoBRL). Samples were denatured at $95^{\circ} \mathrm{C}$ for $5 \mathrm{~min}$ followed by 30 cycles of $95^{\circ} \mathrm{C}$ for $1 \mathrm{~min}, 65^{\circ} \mathrm{C}$ for $1.5 \mathrm{~min}$ and $72{ }^{\circ} \mathrm{C}$ for $1 \mathrm{~min}$, and a final extension at $72^{\circ} \mathrm{C}$ for $10 \mathrm{~min}$. The $0.8 \mathrm{~kb}$ product was purified on a Qiagen PCR purification column, digested with BamHI, purified again and cloned in pBluescript II SK $(+)$. Convergent overlapping sequences were obtained from standard primers contained on the vector. All mutations were verified by sequencing at least two independently generated PCR products. Sequences were analysed using the Wisconsin GCG package. 


\section{RESULTS AND DISCUSSION}

\section{Isolation and phenotypic characterization of revertant strains}

The $\mathrm{Abs}^{-}$phenotype of the absA mutant strains C542 and C577 is characterized by a failure to produce any of the known $S$. coelicolor antibiotics. Cultures grown on R5 agar therefore lack the blue, purple or brick (depending on $\mathrm{pH}$ ) coloration typical of the parental J1501, but nevertheless form a sporulating aerial mycelium much like that of J1501.

While culturing $\mathrm{Abs}^{-}$strains, we observed that both C542 and C577 appeared to revert spontaneously, acquiring pigmented sectors that were stably culturable. These could be sorted into two phenotypic groups: Type I produced a colony very similar to that of the J1501 parent in both pigmentation and morphology; Type II produced a colony that was very deeply pigmented and also was altered in morphology, with a crenulated surface and few aerial hyphae. The crenulation was especially noticeable in colonies grown at low density. Both C542 and C577 produced the two types of pigmented strains.

The frequency of phenotypic reversion in C542 and C577, although not quantified, appeared to be much higher than for other $S$. coelicolor non-antibioticproducing mutants, including abs $B$, bld $B$, bld $G$, bld $H$ (W. Champness, unpublished) and bldA (Guthrie et al., 1998; W. Champness, unpublished). By 2 weeks, many abs A mutant colonies contained a pigmented sector, whereas cultures of the latter set of mutants typically contained only a few pigmented sectors per plate. Moreover, the pigmented sectors that arose spontaneously in $a b s B$ and bld mutants were mostly of the $\mathrm{Cm}^{\mathrm{s}}$ (chloramphenicol-sensitive), 'scarlet' type (Sermonti et al., 1977), a pigmentation phenotype associated with DNA rearrangements (Dyson \& Schrempf, 1987) but otherwise poorly understood. In contrast, the apparent revertant strains isolated from C542 and C577 were as $\mathrm{Cm}^{\mathrm{r}}$ as J1501 (data not shown).

Four Type I and seven Type II pigmented strains (Table 1 ), all independently isolated, were evaluated in plategrown cultures for production of the $S$. coelicolor antibiotics Act and Red. Each strain produced both antibiotics. All strains except C577S20 and C577S25 produced the CDA. An SCP1 $1_{\mathrm{NF}}$ strain, C5422S1, produced Mmy. A notable characteristic of the Type II strains was that they produced Act (especially $\gamma$ actinorhodin; Bystrykh et al., 1996) and Red about $1 \mathrm{~d}$ earlier than did J1501. Below we refer to this Type II phenotype as Pha (precocious hyperproduction of antibiotics).

\section{Genetic evidence for second-site suppressor mutations}

For initial genetic characterization, Pha Type II strain C542S11 was chosen; spores were purified by acid treatment (see Methods) designed to kill any mycelial (multi-genomic) fragments and ensure genetic homogeneity.

To distinguish whether restoration of antibiotic production was due to a reverting mutation or to a secondsite suppressing mutation, strain C542S11 was crossed to a standard mapping strain, J650, and progeny were examined for the $\mathrm{Abs}^{-}$phenotype, occurrence of which would indicate that C542S11 carried both the absA1-542 mutant site and a suppressive mutant site, sab (for suppressor of $\underline{a b s}$ ). $\mathrm{Abs}^{-}$colonies were obtained at a frequency of about one in 5000 among $\operatorname{Str}^{\mathrm{r}}$ progeny from the cross. In contrast, $\mathrm{Abs}^{-}$colonies could not be found in a screen of approximately 200000 colonies plated from C542S11 spores. Together, these results suggested that the $\mathrm{Abs}^{-}$colonies arising from the C542S11 $\times \mathrm{J} 650$ cross were likely absA1-542 $s a b^{+}$recombinants. To verify that the recombinants' $\mathrm{Abs}^{-}$phenotypes were due to abs A mutant alleles, several $\mathrm{Abs}^{-}$strains from the C542S11 $\times$ J650 cross were characterized in detail: their phenotypes were identical to that of C542, and genetic crosses demonstrated that each carried a mutant abs $A$ allele (data not shown).

\section{Genetic mapping of sab suppressor mutations}

The recovery of $\mathrm{Abs}^{-}$recombinants from the C542S11 $\times \mathrm{J} 650$ cross was strong evidence for the existence of a sab mutant allele in C542S11, and the very low frequency of $\mathrm{Abs}^{-}$recombinants from the cross suggested that the $s a b$ mutation site was very close to the absA1-542 mutant site. To map the putative sab locus, additional crosses were performed.

(i) Evidence consistent with a sab locus in the 10 o'clock region, where abs $A$ is also located, came from crossing strain C5422S1 with C542 (Table 1). The results are shown in Fig. 1(a). C5422S1 was a Type II sab mutant isolated in a genetic background (C5422 in Table 1) useful for mapping in the vicinity of absA; this strain had the genotype sab absA1-542 cysA15 proA1 $\arg A 1$ strA1 SCP1 $1_{\mathrm{NF}}$. Strain C5422S1 carried SCP1 integrated in the 9 o'clock region, whereas C542 lacked SCP1. The fertility characteristics of this type of cross, referred to as 'NF $\times$ UF' (Hopwood \& Chater, 1974), result in a curious bias in the alleles that are recovered in progeny spores, even in the absence of selection: all progeny inherit the integrated SCP1 plasmid but almost all carry genomic markers from the SCP1- $1^{-}$parent for the region extending clockwise from approximately 11 o'clock to about 7 o'clock. In Fig. 1(a), this effect can be observed for the his $A$ and $\arg A$ loci: $66 / 68$ spores were his $A 1$ $\mathrm{arg}^{+}$. Because 34/68 spores were Pgm ${ }^{+}$(e.g. sab mutant) and the other 34 were $\mathrm{Pgm}^{-}$(e.g. $s a b^{+}$), the $s a b$ locus appeared to be close to $S C P 1_{\mathrm{NF}}$, either between $\mathrm{SCP} 1_{\mathrm{NF}}$ and $c y s A$ or between $\mathrm{SCP} 1_{\mathrm{NF}}$ and $u r a A$. Four additional $s a b$ mutants isolated from strain C5422 gave similar results.

(ii) The cross shown in Fig. 1(b) further refined mapping of the $s a b$ locus to the 10 o'clock region by assessing its linkage to $c y s A$, a marker to which the absA locus is linked (Adamidis et al., 1990). When strains C5422S1 
(a)

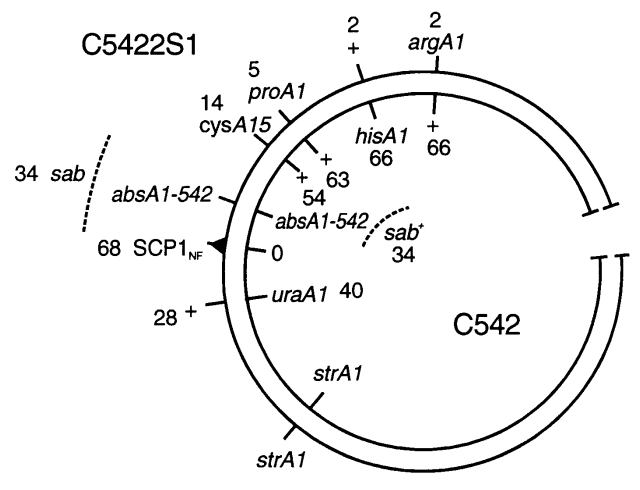

(b)

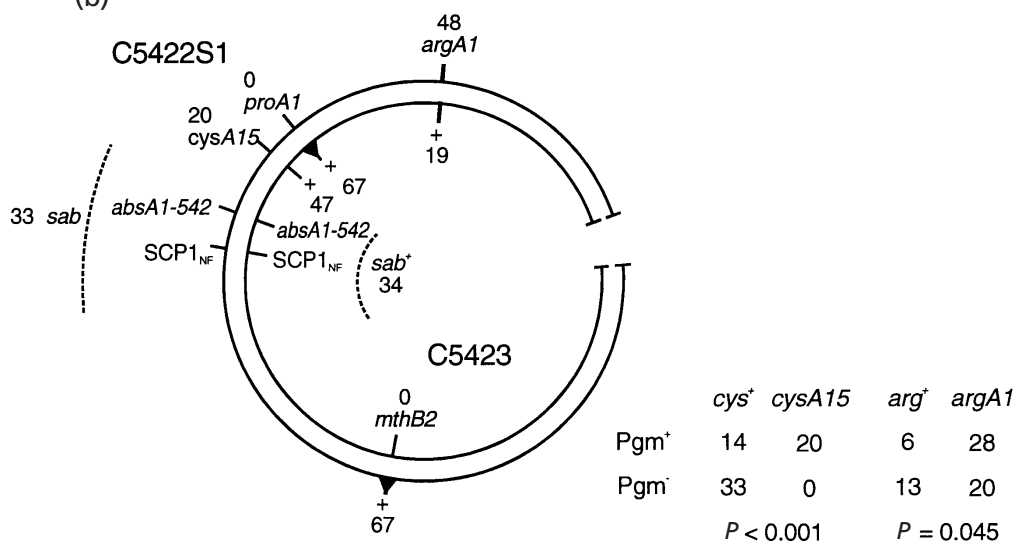

Fig. 1. (a) Cross of strain C5422S1 to parental strain C542. Recombinants were obtained from nonselective plating conditions (see Methods). Allele frequencies among the progeny are indicated. (b) Cross of pseudorevertant strain C5422S1 to strain C5423. Recombinants were selected as indicated by triangles. Allele frequencies among the recombinants are indicated. Segregation of $\mathrm{Pgm}^{+}$and $\mathrm{Pgm}^{-}$indicating the sab mutant and sab alleles, respectively, is tabulated.

(a)

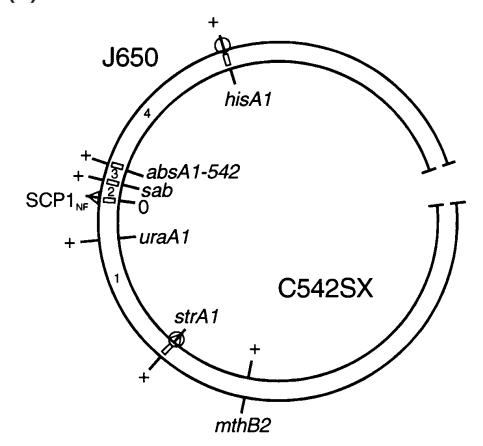

Percentage $\mathrm{Abs}^{-}$with selection for:

$\begin{array}{lcc} & 1 & 2 \\ \text { sab strain } & \text { strA1SCP1 }_{\mathrm{NF}} & \text { strA1his } \mathrm{SCP}_{\mathrm{NF}} \\ \text { C542S2 } & 0.01 & \mathrm{ND} \\ \text { C542S3 } & 0.03 & 0.0025 \\ \text { C542S9 } & 0.01 & \mathrm{ND} \\ \text { C542S10 } & 0.02 & 0.0025 \\ \text { C542S11 } & 0.04 & \mathrm{ND}\end{array}$

(b)

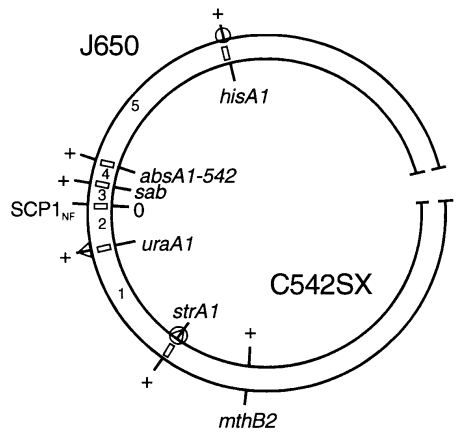

Percentage $\mathrm{Abs}^{-}$with selection for:

$\begin{array}{ccc} & 1 & 2 \\ \text { sab strain } & \text { strA1ura }{ }^{+} \mathrm{SCP} 1_{\mathrm{NF}} & \text { strA1his } \mathrm{SCP}_{\mathrm{NF}} \\ \text { C542S3 } & 0.5 & 0.6 \\ \text { C542S9 } & 3.2 & 0.07\end{array}$

C542S9

3.2

0.07
Fig. 2. Mapping sab alleles. (a) Strain $J 650$ was crossed with the sab strains listed. The percentage of recombinants that were $\mathrm{Abs}^{-}$ is tabulated for two selections. In selection (1) Str $^{r}$ recombinants were selected (indicated by triangles); $\mathrm{SCP} 1_{\mathrm{NF}}$ is also inherited by most progeny (see Methods). In selection (2) $\mathrm{His}^{+} \mathrm{Str}^{r}$ recombinants were selected (indicated by circles). Again, most progeny inherited SCP1 $1_{\mathrm{NF}}$. (b) Protoplast fusion of strains $\mathrm{J} 650$ and $s a b$ strains C542S3 and C542S9. The percentage of recombinants that were $\mathrm{Abs}^{-}$is tabulated for two selections. In selection (1), Ura ${ }^{+} \mathrm{Str}^{\mathrm{r}}$ recombinants were selected (indicated by triangles); in selection (2), $\mathrm{His}^{+} \mathrm{Str}^{\mathrm{r}}$ recombinants were selected (indicated by circles). and C5423 (Table 1) were crossed, the sab allele segregated with the cys 15 allele and lay anticlockwise to it, as had been previously observed for abs A1-542 (Adamidis et al., 1990).

(iii) Crosses like that shown in Fig. 2(a) mapped additional $s a b$ mutations in some of the $s a b$ strains listed in Table 1. Before being used in crosses, each strain was purified as described in Methods, and its phenotypic stability was evaluated: no $\mathrm{Abs}^{-}$colonies were found among approximately 200000 colonies screened by microscopic analysis of plates containing several thousand colonies each.
The $s a b$ strains were crossed against J650 (an antibioticproducing strain) and the recombinant progeny were evaluated for the $\mathrm{Abs}^{-}$phenotype. Spores were first selected for the presence of the strA1 allele. All of these progeny also inherited $\mathrm{SCP} 1_{\mathrm{NF}}$ as well as the J650 9 o'clock region (because the parental plasmid status was $\mathrm{NF} \times \mathrm{UF}$, as discussed above). Thus, all progeny genomes were recombinant in interval 1 . A second selection scheme imposed a requirement for the $\mathrm{his}^{+}$ allele as well as for $\operatorname{str} A 1$. With this selection, two $\mathrm{Abs}^{-}$ colonies were found among over $100000 \mathrm{His}^{+} \mathrm{Str}^{+}$ colonies from each of the C542S10 and C542S3 crosses. In contrast, the first selection scheme yielded $\mathrm{Abs}^{-}$ 
colonies at 10-fold higher frequencies. These results suggested the relative order of the $s a b$ and $a b s A$ alleles drawn in Fig. 2(a): $\mathrm{Abs}^{-}$colonies could result from crossovers in intervals 1 and 3 in the first selection for Str ${ }^{\mathrm{r}}$, but would require more crossovers, in intervals 1,3 and 4, for the $\mathrm{His}^{+} \mathrm{Str}^{\mathrm{r}}$ selection.

\section{A sab locus maps close to the absA locus}

As tabulated in Fig. 2(a), crosses with the pseudorevertant strains all yielded a low percentage of $\mathrm{Abs}^{-}$ progeny. Difficulty in detecting the infrequent $a b s A 1$ $s a b^{+}$recombinants precluded making significant distinctions between the locations of the various $s a b$ mutations. Because protoplast fusions are potentially capable of increasing the accuracy of recombination analysis due to the higher recombination frequency in such crosses (Hopwood et al., 1977), we explored this procedure. As in $\mathrm{NF} \times \mathrm{UF}$ plasmid-mediated crosses, $\mathrm{SCP} 1_{\mathrm{NF}} \times \mathrm{UF}$ protoplast fusions typically result in acquisition of SCP1 ${ }_{\mathrm{NF}}$ in all progeny (Hopwood et al., 1977). However, in contrast to $\mathrm{NF} \times \mathrm{UF}$ matings, the frequency of unselected parental markers at other loci is not biased toward either parental genotype.

Protoplasts of $s a b$ strains C542S3 or C542S9 were mixed with $\mathrm{J} 650$ protoplasts and recombinant progeny were analysed. Two classes of recombinants were selected: $\mathrm{Str}^{\mathrm{r}} \mathrm{Ura}^{+}$and $\mathrm{Str}^{\mathrm{r}} \mathrm{His}^{+}$. Among these, $\mathrm{Abs}^{-}$colonies were found at higher frequencies than in plasmidmediated crosses, as predicted (e.g. $0.6 \%$ vs $0.0025 \%$ for the strA1 his $^{+} \mathrm{SCP}_{\mathrm{NF}}$ selection with strain C542S3). Mock protoplast fusions using only the individual sab strains, without J650, yielded no $\mathrm{Abs}^{-}$colonies.

The recombination data for C542S9 suggested the relative order for the $s a b$ and $a b s A$ alleles shown in Fig. 2 (b). This order would be consistent with the higher frequency of $\mathrm{Abs}^{-}$recombinants found in the $\mathrm{Str}^{\mathrm{r}} \mathrm{Ura}^{+}$ selection compared to the $\operatorname{Str}^{\mathrm{r}} \mathrm{His}^{+}$selection: in the former, two recombination events, in intervals 1 and 4, could produce $\mathrm{Abs}^{-}$progeny whereas in the latter, three recombination events, in intervals 1 or 2,4 and 5 , would be necessary. The data for C542S3, however, showed no significant difference in recombinant frequencies for the two selections. This observation is discussed further below.

The observed frequencies of recombination between the $s a b$ and absA1 alleles did not provide sufficient information to accurately determine their physical distances, and therefore to suggest whether sab alleles were intragenic or extragenic. Very little data correlating recombination frequencies with physical distance are available for streptomycetes. To make one such comparison, we measured the recombination frequency between mutations in the act gene cluster, which has been completely cloned and sequenced. Using protoplast fusions of strains TK16 and TK18 (Table 1), we obtained $3.7 \%$ act ${ }^{+}$recombinants between the act-141 and act117 mutations, which affect the actIII and actIV loci, respectively, and so the mutations would be approximately $5 \mathrm{~kb}$ apart in the act cluster (Malpartida \&

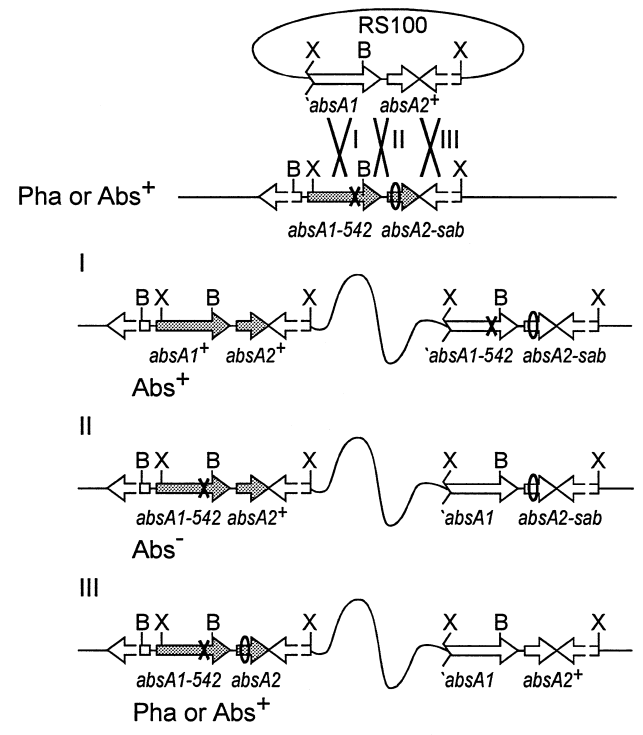

$\begin{array}{cc}\text { Strain } & \begin{array}{c}\text { Frequency of Abs } \\ \text { RS100 lysogens }\end{array} \\ \text { C542S2 } & 2 / 285 \\ \text { C542S3 } & 3 / 14 \\ \text { C542S9 } & 24 / 78 \\ \text { C542S10 } & 3 / 75\end{array}$

Fig. 3. Mapping $s a b$ alleles to a $3.2 \mathrm{~kb}$ Xhol-Xhol interval. RS100 carries absA2 and 5 'truncated absA1 gene (indicated by 'absA1). The absA1-542 mutation ( $\mathbf{X}$ ) has been mapped to absA1 (Brian et al., 1996). The sab mutation (0) is hypothesized to be in absA2. If the sab mutant site is covered by the RS100 insert, recombination in interval II, between the two mutant sites, could produce $\mathrm{Abs}^{-}$lysogens. Shading indicates the expressed copy of absA. B, BamHI; X, Xhol.

Hopwood, 1986). Considering these data with respect to the $s a b$-abs $A 1$ recombination frequencies suggested that the $s a b$ mutant sites could lie within the same gene as the abs A1-542 mutation or very close by.

\section{Isolation of sab alleles on specialized transducing phage}

The mapping results for the $s a b$ mutations suggested that they might be close enough to abs A to lie within the region carried by an $11 \mathrm{~kb}$ abs A1-542-complementing clone, pWC3151, that we had obtained in other experiments (Brian et al., 1996). Accordingly we subcloned DNA from pWC3151 in a temperate phage vector, KC516, for marker rescue experiments. To establish lysogens in $a b s A 1-542 s a b$ strains, we used phage RS100, which carries a $3 \cdot 2 \mathrm{~kb}$ XhoI fragment (Fig. 5a) spanning the absA1 mutant sites (Brian et al., 1996), and also including $a b s A 2$. Fig. 3 illustrates the genotypes expected in such lysogens. In this example, a sab mutant site is hypothesized in absA2 (see below) and three intervals for recombination are delineated. Two considerations are important in interpreting the lysogen phenotypes. First, the RS100 cloned fragment lacks the absA1 N- 

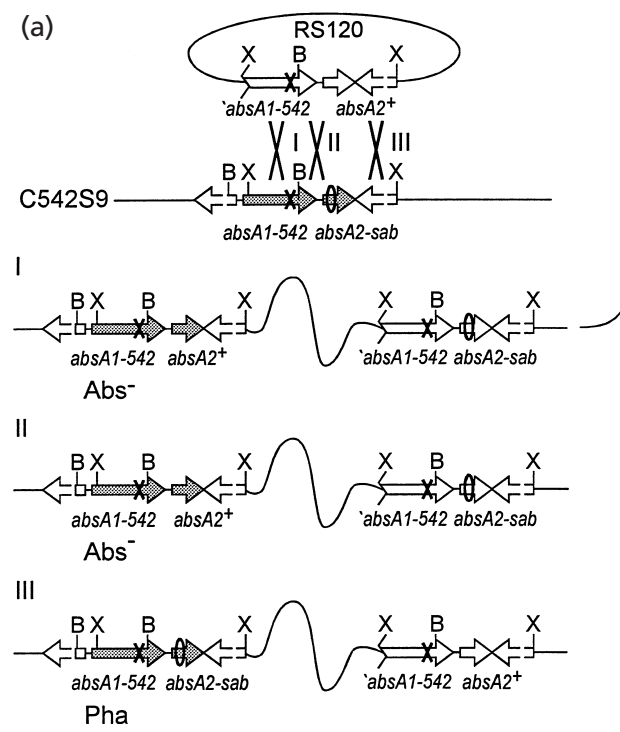

(b)

II
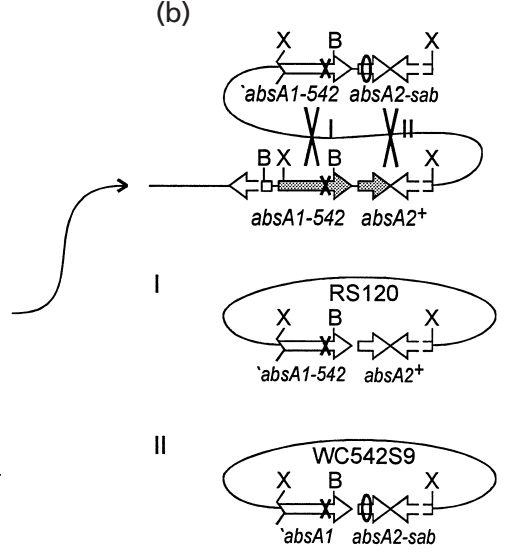

(c)
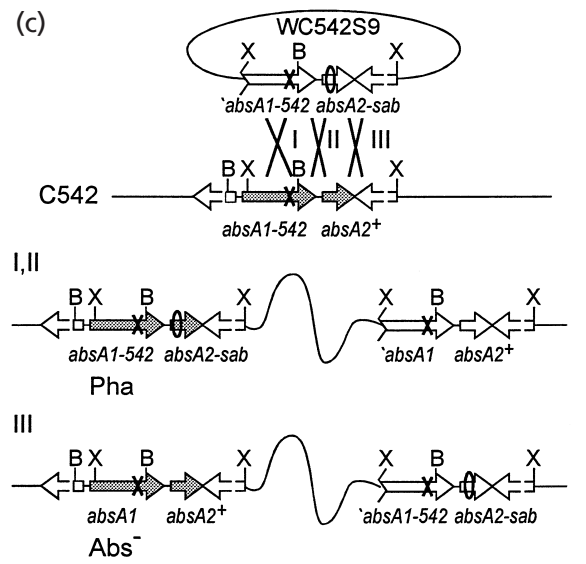

Fig. 4. Crossing the sab9 mutation onto the phage-cloned insert. RS120 carries the absA1-542 mutation in the 5'truncated absA1 gene. (a) Recombination in intervals I or II could produce Abs ${ }^{-}$lysogens. (b) Released phage could carry the sab9 mutation. (c) A phage strain, WC542S9, could transduce C542 to the Pha phenotype. B, BamHI; X, Xhol.

terminus and transcription start site; second, abs 11 and abs 22 are predicted to be cotranscribed (T. Anderson, unpublished). Lysogens, therefore, would contain one expressed copy of the absA locus and one non-expressed copy; those formed by recombination in interval II would carry the $s a b$ mutant site in the non-expressed copy and the absA1-542 $s a b^{+}$alleles in the expressed copy. Hence, recovery of $\mathrm{Abs}^{-}$lysogens following infection of a $s a b$ strain by RS100 would indicate that the sab mutant site was covered by the cloned XhoI fragment. For four strains shown in Fig. 3, recovery of $\mathrm{Abs}^{-}$lysogens following this protocol indicated that the $s a b$ mutant sites were located within the $3.2 \mathrm{~kb} X h o \mathrm{I}$ interval; for C542S9, the result was confirmed by marker exchange, recovering the $s a b$ allele on the cloned $3 \cdot 2 \mathrm{~kb}$ XhoI fragment.

For marker exchange experiments, the protocol illustrated in Fig. 4 was used. The cloned XhoI fragment used to recover the $s a b$ allele carried the absA1-542 mutation. This fragment was cloned in phage RS120 (Fig. 5a; Brian et al., 1996) and lysogens were established; Fig. 4(a) shows the genotypes of lysogens established in the Pha strain C542S9. Phage recovered from lysogens (Fig. 4b) were plaque-purified and tested for transduction of the Pha phenotype to C542 (Fig. 4c). As shown, phage WC542S9 was able to transduce the sab9 allele from C542S9 to C542, creating Pha lysogens. This result confirmed that the sab9 mutation lay in the $3.2 \mathrm{~kb}$ XhoI interval and also indicated that $s a b 9$ was sufficient to create the Pha phenotype.

\section{Epistasis relationships of absA mutant alleles}

The sab9 mutation's close linkage to absA1-542 raised the possibility that it might lie within the absA1 or absA2 genes. Additional evidence supporting such a notion came from abs A mutants' epistasis relationships, which, as discussed below, predicted that certain mutations in absA1 or absA2 might suppress the $\mathrm{Abs}^{-}$ phenotype of C542.

In previous genetic studies, two kinds of disruption mutations in absA caused a Pha sab-Type II-like phenotype (Brian et al., 1996; Aceti \& Champness, 1998). These mutations (Fig. 5a) were (1) an ermE insertion/deletion in strain C420, eliminating much of the absA1 gene and probably also expression of the downstream absA2 gene, and (2) an insertional disruption (in strain C430) created by single-crossover integration of a phage-cloned fragment internal to the abs A transcript (phage RS500 in Fig. 5a). The latter mutation primarily disrupted absA2 expression; it also truncated the C-terminus of absA1, but complementation results suggested that the truncated AbsA1 protein was active in vivo. Because both disruptions eliminated absA2 function, we proposed (Brian et al., 1996) that AbsA2 functions as a negative regulator of antibiotic synthesis.

The abs $A$ alleles that cause an $\mathrm{Abs}^{-}$phenotype mapped to absA1. One hypothesis for their effects is that they lock the abs A two-component system into a negatively regulating mode. Disruption of absA2 might thus be epistatic to the absA1-542 and absA1-577 mutations. The Pha phenotype of RS500 lysogens established in C542 and C577 (see Methods) confirmed this prediction.

\section{Sequence analysis of $\mathrm{Abs}^{-}$mutant strains}

In previous work (Brian et al., 1996), marker rescue experiments were used to localize absA mutations responsible for the $\mathrm{Abs}^{-}$phenotype to the XhoI-BamHI interval within absA1 (Fig. 5a). To define the mutant 
(a)

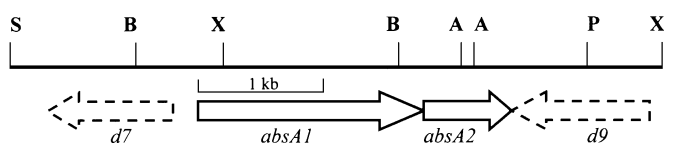

RS100/RS120

$\mathbf{X}$

RS500

C420
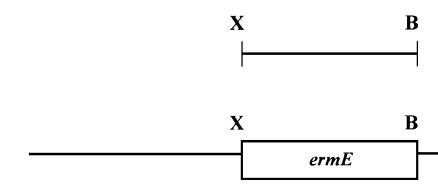

(b)

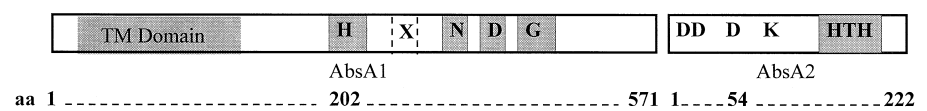

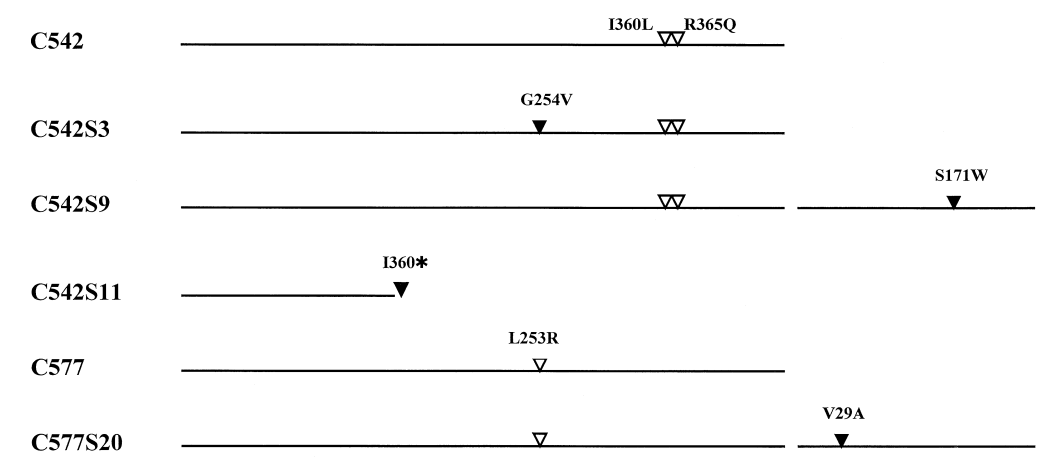

Fig. 5. (a) Map of the absA locus. The absA1 gene encodes a histidine kinase sensortransmitter; absA2 encodes a response regulator. $d 7$ and $d 9$ are ORFs which are predicted to be part of the CDA gene cluster (www.sanger.ac.uk/pub/s_coelicolor/ sequence). Phage strains RS100, RS120 and RS500 (Table 1) contain the inserts shown; C420 (Table 1) contains the ermE replacement shown. (b) Mutational alterations to the AbsA 1 and AbsA2 proteins in absA1 (open triangle) and sab (filled triangle) mutant strains. Conserved domains (see text) in two-component systems (Parkinson, 1995; Stock et al., 1995) are indicated. A, Apal; B, BamHI; P, Pstl; S, Sacl; $\mathrm{X}$, Xhol. sites present in strains C542 and C577, the absA1 gene was sequenced.

The absA1 mutations found in C542 and C577 are shown in Fig. 5(b). C577 contained a single amino acid change, L253R. C542 contained two amino acid changes (I360L and R365Q). We do not know if both mutations in $\mathrm{C} 542$ are required to cause the $\mathrm{Abs}^{-}$phenotype. Sequencing absA2 in C542 and C577 confirmed that in these strains abs 22 contained no mutations.

The C542 and C577 mutations lie in regions of AbsA1 that are conserved in the histidine kinase sensortransmitter family: the C542 mutations alter the ' $G$ box', which is involved in nucleotide binding (Stock et al., 1995), and the C577 mutation alters a region, provisionally named the ' $\mathrm{X}$ box', and recently proposed to be involved in the aspartylphosphatase activity common to many sensor-transmitters (Hsing et al., 1998).

\section{Sequence analysis of sab mutations}

To locate $s a b$ mutations potentially within the abs $A$ locus, we amplified regions of the $a b s A 1$ and $a b s A 2$ genes by PCR, and then sequenced the products from two independent amplifications of each mutant strain.
Fig. 5(b) shows the sab mutant sites found in this analysis.

$a b s A 2$ was sequenced in $10 s a b$ strains, including those genetically analysed in the experiments discussed above. In C542S9, sequencing revealed an S171W change at the $\mathrm{N}$-terminal end of the predicted helix-turn-helix region.

In one $s a b$ strain, C577S25, the $a b s A$ locus had undergone a deletion that started within the abs 11 gene and extended through abs $A 2$ into the neighbouring ORF, $d 9$ (Fig. 5a).

Both C542S9 and C577S25 were Type II sab strains with a Pha phenotype. Interestingly, C542S9 was even more strongly pigmented than C577S25 and produced more $\gamma$ actinorhodin than any other sab strain. This was surprising because if the $a b s A$ system functions as a negative regulator, the phenotype of a $\Delta a b s A 1 a b s A 2$ mutation might be expected to overproduce more strongly than an abs 22 point mutation. Thus this result hints at a complex role for the AbsA2 response regulator in antibiotic production. Another possibility is that deletion of the neighbouring uncharacterized DNA modulates the Pha effect in C577S25.

One Type I strain, C577S20, contained an absA2 mutation, V29A, that appeared to restore at least some 
'normal' AbsA function. It did not alter any of the domains known to be involved in phosphotransfer reactions but affected a region that was expected from the NarL response regulator crystal structure (Baikalov et al., 1996) to be $\alpha$-helical.

As mentioned above, the strains C577S20 and C577S25 were $\mathrm{CDA}^{-}$. For C577S25, this can be explained by the observation that the $s a b$ mutation is a deletion which extends into a $c d a$ biosynthetic region (Fig. 5a, b). For C577S20 we do not have an explanation for the $\mathrm{CDA}^{-}$phenotype at this time.

No abs 22 mutations were found in the other strains sequenced. However, the marker rescue experiments discussed above had strongly predicted that the $s a b$ alleles in the strains tested lay in the $3 \cdot 2 \mathrm{~kb} X h o I$ interval (Fig. 3), and it was possible that absA1 could be mutant in some sabs. Therefore, we sequenced absA1 in two strains: C542S3, a Type I strain that had been used in all of the genetic experiments; and C542S11, a Type II strain used in initial investigations of the pseudoreversion phenomenon. Both strains proved to contain $s a b$ mutations in absA1, as well as in the absA1-542 mutations discussed above.

Strain C542S3 contained the mutation G252V. This amino acid is in the same region of AbsA1 in which the $\mathrm{Abs}^{-}$strain C577 is mutant. In fact, the two mutations affect adjacent residues, posing the questions about whether the sab3 mutation could suppress the absA1577 mutation and whether the abs A1-577 mutation could suppress the absA1-542 mutation. However, we have not yet tested these possibilities or otherwise addressed whether the various $s a b$ mutations are allelespecific.

The Type II strain C542S11 contained an absA1 nonsense mutation in amino acid residue 360 . The Pha phenotype may have been due to a polar effect on absA2 expression. Alternatively, if the nonsense mutation was not polar, the antibiotic might be overproduced because phosphorylation of AbsA2 is necessary for its negatively regulating function, and the mutant lacks AbsA1 kinase activity.

\section{Do sab mutations occur outside the absA locus?}

Two of $10 s a b$ strains in which $a b s A 2$ was sequenced contained absA2 mutations and two more strains in which absA1 was sequenced contained absA1 mutations. Of the remaining strains, two (C542S2 and C54210) were used in the marker rescue mapping experiments that predicted close linkage of the absA1542 and $s a b$ alleles. Thus, these would be expected to contain mutations in absA1, but could contain mutations in the $2.7 \mathrm{~kb}$ uncharacterized region (rightwards of absA2 in Fig. 5a) cloned in phage RS100 [however, the relatively low recombination frequency of $\mathrm{Abs}^{-}$lysogens (Fig. 3) compared to the sab9 mutation in $a b s A 2$ argues against this possibility]. Altogether, $11 s a b$ strains have been used in one of the types of crosses illustrated in Figs 1-3 and in all cases there was evidence for a sab mutation close to absA. Nevertheless, the analysis discussed here does not exclude the possibility that $s a b$ mutations could occur outside absA.

\section{Correlation of mapping data with sequencing data and the physical map}

The protoplast fusion results with strain C542S9 led us to predict in Fig. 2(b) that the relative order for absA1542 and $s a b$, with respect to other genetic markers, would be (in anticlockwise order), hisA-absA1-sab$u r a A$. Considering that $s a b 9$ lies in absA2, this would give a relative gene order for $a b s A 1$ and $a b s A 2$ of bisA-absA1-absA2-uraA. We have located the absA locus on the ordered cosmid E8 (Redenbach et al., 1996), and the order has been confirmed during the ongoing genomic sequencing (www.sanger.ac.uk/pub/ s_coelicolor/sequence).

The protoplast fusion results (Fig. 2b) did not predict a relative order for the $a b s A 1$ and sab3 mutations in strain C542S3, since there was no significant difference in the frequency of $a b s A 1-542 s a b^{+}$recombinants for the two selections. In light of the close linkage of absA1-542 and $s a b 3$ in the abs 11 sequence (Fig. $5 \mathrm{~b}$ ), gene conversion events could have contributed significantly to production of recombinant genotypes, and so distorted apparent recombination frequencies. The marker rescue experiment in Fig. 3 and the plasmid-mediated recombination experiment in Fig. 2 would also be subject to gene conversion effects that could affect ordering of mutations that are close together.

\section{Conclusions}

(i) Non-antibiotic-producing $\left(\mathrm{Abs}^{-}\right)$mutants of the abs $A$ locus, which seem to lock the AbsA regulatory system into a negatively regulating mode, contain point mutations in conserved domains of the AbsA1 histidine kinase sensor-transmitter protein.

(ii) The absA1 mutants spontaneously acquire suppressive mutations that restore antibiotic synthesis.

(iii) Plasmid-mediated and protoplast fusion mapping techniques were useful for genetic analysis of suppressive $(s a b)$ mutations, locating some close to abs A.

(iv) Actinophage $\phi \mathrm{C} 31$-derived vectors were useful for marker rescue and marker exchange experiments that verified the existence and location of $s a b$ mutations and allowed transduction of $s a b$ mutations from strain to strain.

(v) Sequence analysis defined $s a b$ mutant residues in the abs A two-component system. Some sab alleles (Type I) restore apparently normal AbsA function since they restore a wild-type phenotype to $\mathrm{Abs}^{-}$mutants, whereas some (Type II) cause antibiotic overproduction.

(vi) Antibiotic overproduction in $s a b$ strains can result from deletion of absA, consistent with absA's proposed role as a negative regulator, but the most strongly pigmented $s a b$ strain contains a point mutation in the 
AbsA2 response regulator, suggesting a complex role for the abs $A$ locus in production of antibiotics.

\section{ACKNOWLEDGEMENTS}

We thank Maryuska Calderón for technical assistance and Heather Bobier and Tom Lucier for help with mapping experiments. This work was supported by grants MCB9604055 and MCB9206068 from the National Science Foundation to W.C.

\section{REFERENCES}

Aceti, D. \& Champness, W. (1998). Transcriptional regulation of Streptomyces coelicolor pathway-specific antibiotic regulators by the $a b s A$ and $a b s B$ loci. J Bacteriol 180, 3100-3106.

Adamidis, T., Riggle, P. \& Champness, W. (1990). Mutations in a new Streptomyces coelicolor locus which globally block antibiotic biosynthesis but not sporulation. J Bacteriol 172, 2962-2969.

Baikalov, I., Schroder, I., Kaczor-Grzeskowiak, M., Grzeskowiak, K., Gunsalus, R. P. \& Dickerson, R. E. (1996). Structure of the Escherichia coli response regulator NarL. Biochemistry 35, 11053-11061.

Bibb, M. (1996). The regulation of antibiotic production in Streptomyces coelicolor A3(2). Microbiology 142, 1335-1344.

Brian, P., Riggle, P. J., Santos, R. A. \& Champness, W. C. (1996). Global negative regulation of Streptomyces coelicolor antibiotic synthesis mediated by an absA-encoded putative signal transduction system. J Bacteriol 178, 3221-3231.

Bystrykh, L. V., Fernández-Moreno, M. A., Herrema, J. K., Malpartida, F., Hopwood, D. A. \& Dijkhuizen, L. (1996). Production of actinorhodin-related blue pigments by Streptomyces coelicolor A3(2). J Bacteriol 178, 2238-2244.

Chakraburtty, R. \& Bibb, M. (1997). The ppGpp synthetase gene (relA) of Streptomyces coelicolor A3(2) plays a conditional role in antibiotic production and morphological differentiation. $J$ Bacteriol 179, 5854-5861.

Champness, W. C. (1988). New loci required for Streptomyces coelicolor morphological and physiological differentiation. J Bacteriol 170, 1168-1174.

Champness, W. (1999a). Cloning and analysis of regulatory genes involved in Streptomyces secondary metabolite biosynthesis. In Manual of Industrial Microbiology and Biotechnology, 2nd edn, pp. 725-739. Edited by A. Demain \& J. Davies. Washington, DC: American Society for Microbiology.

Champness, W. (1999b). Actinomycete phylogeny and development: questions and challenges. In Prokaryotic Development. Edited by L. Shimkits \& Y. Brun. Washington, DC: American Society for Microbiology (in press).

Champness, W. \& Chater, K. (1994). Regulation and integration of antibiotic production and morphological differentiation in Streptomyces spp. In Regulation of Bacterial Differentiation, pp. 61-94. Edited by P. Piggot, C. Moran \& P. Youngman. Washington, DC: American Society for Microbiology.

Chater, K. F. \& Bibb, M. J. (1997). Regulation of bacterial antibiotic production. In Biotechnology, Vol. 6, Products of Secondary Metabolism, pp. 57-105. Edited by H. Kleinkauf \& H. von Döhren. Weinheim: VCH.

Chater, K. F. \& Bruton, C. J. (1985). Resistance, regulatory and production genes for the antibiotic methylenomycin are clustered. EMBO J 4, 1893-1897.

Chong, P. P., Podmore, S. M., Kieser, H. M., Redenbach, M., Turgay, K., Marahiel, M., Hopwood, D. A. \& Smith, C. P. (1998).
Physical identification of a chromosomal locus encoding biosynthetic genes for the lipopeptide calcium-dependent antibiotic (CDA) of Streptomyces coelicolor A3(2). Microbiology 144, 193-199.

Dyson, P. \& Schrempf, H. (1987). Genetic instability and DNA amplification in Streptomyces lividans 66. J Bacteriol 169, 4796-4803.

Fernández-Moreno, M. A., Cabellero, J. L., Hopwood, D. A. \& Malpartida, F. (1991). The act gene cluster contains regulatory and antibiotic export genes, direct targets for translational control by the bldA tRNA gene of Streptomyces coelicolor. Cell 66, 769-780.

Gramajo, H. E., Takano, E. \& Bibb, M. J. (1993). Stationary phase production of the antibiotic actinorhodin in Streptomyces coelicolor A3(2) is transcriptionally regulated. Mol Microbiol 7, 837-845.

Guthrie, E., Flaxman, C., White, J., Hodgson, D. A., Bibb, M. J. \& Chater, K. F. (1998). A response-regulator-like activator of antibiotic synthesis from Streptomyces coelicolor A3(2) with an amino-terminal domain that lacks a phosphorylation pocket. Microbiology 144, 727-738.

Hodgson, D. \& Chater, K. (1981). A chromosomal locus controlling extracellular agarase produced by Streptomyces coelicolor A3(2) and its inactivation by chromosomal integration of plasmid SCP1. J Gen Microbiol 124, 339-348.

Hopwood, D. \& Chater, K. F. (1974). Streptomyces coelicolor. In Handbook of Genetics, vol. 1, pp. 237-255. Edited by R. C. King. New York: Plenum.

Hopwood, D., Harold, R., Vivian, A. \& Ferguson, H. (1969). A new kind of fertility variant in Streptomyces coelicolor. Genetics 62, 461-477.

Hopwood, D., Wright, H., Bibb, M. \& Cohen, S. (1977). Genetic recombination through protoplast fusion in Streptomyces. Nature 268, 171-174.

Hopwood, D. A., Bibb, M. J., Chater, K. F. \& 7 other authors (1985). Genetic Manipulation of Streptomyces: a Laboratory Manual. Norwich: John Innes Foundation.

Hsing, W., Russo, F. D., Bernd, K. K. \& Silhavy, T. J. (1998). Mutations that alter the kinase and phosphatase activities of the two-component sensor EnvZ. J Bacteriol 180, 4538-4546.

Kirby, R. \& Hopwood, D. (1977). Genetic determination of methylenomycin synthesis by the SCP1 plasmid of Streptomyces coelicolor A3(2). J Gen Microbiol 98, 239-252.

Malpartida, F. \& Hopwood, D. A. (1986). Physical and genetic characterization of the gene cluster for the antibiotic actinorhodin in Streptomyces coelicolor A3(2). Mol Gen Genet 203, 66-73.

Narva, K. E. \& Feitelson, J. S. (1990). Nucleotide sequence and transcriptional analysis of the redD locus of Streptomyces coelicolor A3(2). J Bacteriol 172, 326-333.

Parkinson, J. (1995). Genetic approaches for signaling pathways and proteins. In Two-component Signal Transduction, pp. 9-24. Edited by J. A. Hoch \& T. J. Silhavy. Washington, DC: American Society for Microbiology.

Piret, J. M. \& Chater, K. F. (1985). Phage-mediated cloning of bldA, a region involved in Streptomyces coelicolor morphological development, and its analysis by genetic complementation. J Bacteriol 163, 965-972.

Redenbach, M., Kieser, H. M., Denapaite, D., Eichner, A., Cullum, J., Kinashi, H. \& Hopwood, D. A. (1996). A set of ordered cosmids and a detailed genetic and physical map for the $8 \mathrm{Mb}$ Streptomyces coelicolor A3(2) chromosome. Mol Microbiol 21, 77-96.

Rodicio, M. R., Bruton, C. J. \& Chater, K. F. (1985). New derivatives 
of the Streptomyces phage $\phi \mathrm{C} 31$ useful for the cloning and functional analysis of Streptomyces DNA. Gene 34, 283-292.

Rudd, B. A. M. \& Hopwood, D. A. (1979). Genetics of actinorhodin biosynthesis by Streptomyces coelicolor A3(2). J Gen Microbiol 114, 35-43.

Rudd, B. A. M. \& Hopwood, D. A. (1980). A pigmented mycelial antibiotic in Streptomyces coelicolor: control by a chromosomal gene cluster. J Gen Microbiol 119, 333-340.

Sermonti, G., Petris, A., Micheli, M. R. \& Lanfaloni, L. (1977). A factor involved in chloramphenicol resistance in Streptomyces coelicolor A3(2): its transfer in the absence of the fertility factor. J Gen Microbiol 100, 347-353.

Stock, J., Surette, M., Levit, M. \& Park, P. (1995). Two-component signal transduction systems: structure-function relationships and mechanisms of catalysis. In Two-component Signal Trans- duction, pp. 25-52. Edited by J. Hock \& T. Silhavy. Washington, DC: American Society for Microbiology.

Strohl, W. R. (1997). Biotechnology of Antibiotics, 2nd edn. New York: Marcel Dekker.

Takano, E., Gramajo, H. C., Strauch, E., Andres, N., White, J. \& Bibb, M. J. (1992). Transcriptional regulation of the redD transcriptional activator gene accounts for growth-phase-dependent production of the antibiotic undecylprodigiosin in Streptomyces coelicolor A3(2). Mol Microbiol 6, 2797-2804.

Wietzorrek, A. \& Bibb, M. (1997). A novel family of proteins that regulates antibiotic production in Streptomyces appears to contain an OmpR-like DNA-binding fold. Mol Microbiol 25, 1183-1184.

Received 8 March 1999; revised 26 May 1999; accepted 2 June 1999. 